\title{
Effective Strategy Formation Models for Inventory Management under the Conditions of Uncertainty
}

\author{
Oleg Anatolyevich Kosorukov ${ }^{1} \&$ Olga Alexandrovna Sviridova ${ }^{1}$ \\ ${ }^{1}$ Department of Mathematical Methods in Economics, Plekhanov Russian University of Economics, Russia \\ Correspondence: Olga Alexandrovna Sviridova, Department of Mathematical Methods in Economics, Plekhanov \\ Russian University of Economics, Russia.
}

Received: December 15, 2014 Accepted: January 18, 2015 Online Published: June 28, 2015

doi:10.5539/ies.v8n7p64

URL: http://dx.doi.org/10.5539/ies.v8n7p64

\begin{abstract}
The article deals with the problem of modeling the commodity flows management of a trading company under the conditions of uncertain demand and long supply. The Author presents an analysis of modifications of diversified inventory management system with random demand, for which one can find the optimal inventory control strategies, including those taking into account the time value of money and various limitations, thus maximizing the total cash flow. The Author considers stochastic simulation and optimization models which allow a company to reduce the costs of inventory management.
\end{abstract}

Keywords: inventory management, optimization, simulation modeling

\section{Introduction}

In an increasingly competitive environment on the consumer market, stability of commercial enterprises is provided through the implementation of strategies aimed at meeting the consumer demand not only for commodities, but also for the related services, as well as the reduction of all types of trade costs.

Formation of such strategies predetermines the need to improve various enterprise systems, including inventory management system, based on the rationalization and optimization of commodity flows using the models and methods, appropriate to definition of concerned problems.

The problem of optimizing the inventory level is not new. There are a lot of traditional and well-known models (Wilson EOQ Model or Wilson Formula, and periodic model), their modifications (Lukinskiy, 2007), as well as the copyrighted software products (Goldsbi, 2009). The Classical models of inventory management require relatively constant conditions which are quite rare in reality. Also, they use too approximate values for the calculation of the optimal parameters. The majority of methods are based on the average expectative determinate indicators, and do not take into account the uncertainty, associated with inaccurate or incomplete information about demand, supply, and time delays of commodities ordered, as well as the other parameters of the logistic system (Stepanov, 2006).

The tracking of uncertainty in demand and delivery time, when modeling the inventory management system, is a quite challenging task (Prosvetov, 2008). The fact that the parameters of random variables are not constant and have their temporal dynamics or seasonality may cause additional complexity. In this context, it is hardly possible to apply deterministic mathematical models, and the analytic solution of stochastic inventory management models will apparently either be impossible or too time-consuming. Therefore, in such situations it is advisable to involve other methods, such as, for example, the simulation modeling technique (Sergeev, 2008).

\section{Models and Methods}

One of the current research areas in the field of inventory management systems modeling is related to solving a rather a complicated problem of determining the optimal values of the two parameters of inventory control strategy (scope of delivery and delivery assignment time point) and is associated with the development and analysis of the set of multifactor models.

When designing the optimal inventory control strategy for a trade or manufacturing organization, and when constructing the logistics process optimization model, the first question to arise is the question of selection of the factors considered in the model. Generally, depending on the specifics of a certain organization, there is no 
necessity to consider all these factors simultaneously, sometimes some of them are lacking or have a little effect on the logistic process. The factors that are most often considered in the model include the time value of money; limitations on the size of the working capital, warehouse space, number of trucks, and commodities shelf-life; the uncertainty of the supply timing and demand; lending options (subject to credit availability) and the purchase of commodities (prices, minimum lots, payment terms and so on); as well as transportation order specifications (cost, minimum order period and so on) (Kosorukov, 2005).

\subsection{The Model to Determine Simultaneously Optimal Supply Time and Scope of Delivery with No Regard to the} Time Value of Money

The Economic and mathematical model of the inventory management system and its modifications are developed based on the approach, which describes a logistic process by means of outgoing (expenses) and incoming (income) cash flows for each type of commodities. These flows lay basis for creation of criterion function. Desired characteristics are determined through the maximization of this criterion function. Criterion function reflects the total reduced value of cash flow (income and expenses) within a certain time interval.

The Features of inventory control model, described in the present study, may be presented as follows:

- The duration of the implementation period $T$ of produced (on a single occasion or multiple times) stock of commodities is given;

- The stock of commodities is produced on a single occasion at a time $\mathrm{t}=0$, or in multiple times $t_{j}(\mathrm{j}=$

$1, \ldots, \mathrm{k})$;

- Any arbitrary number of type of commodities $\mathrm{m}$ is taken into account $(\mathrm{i}=1, \ldots, \mathrm{m})$; each type of commodities has its stock. Every time, commodities delivery includes all kinds of products analyzed, though order quantity for each type of product is different;

- For the whole period [0; T] there is a certain number of selected trucks $k(j=1, \ldots, k)$ with the given specifications (volume, cargo mass limit, cargo maximum volume, and the transportation cost);

- The demand is represented by a continuous random variable with a given distribution law within the time frame $[0 ; T]$;

- The criterion function includes payments flow, accounting for the proceeds from the commodities sale, the cost of commodities procurement and transportation costs;

- There is no possibility to postpone the actual date of commodities delivery from the fixed time, i.e., there is no ambiguity about the time of delivery;

- It is required to determine the best time moments ${ }^{t_{j}}$ for stock forming (arrival of trucks) and volume $r_{i}^{j}$ of generated stocks.

Initially, we consider the continuous model without considering of the time value of money with limitations made only on the weight and volume of commodities transported by one track. We introduce the following notation:

$T$-the duration of the time period under consideration;

$m$-the number of commodity types $(i=1, \ldots, m)$,

$k$-the exact number of available trucks;

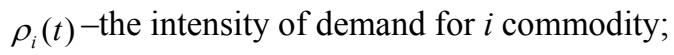

$V$-the limiting volume of cargo carried by one track;

$M$-the limiting mass of cargo carried by one track;

$v_{i}$-the volume of the $i$-thcommodity unit;

$m_{i}$-the weight of the $i$-thcommodity unit;

$c_{i}$-the earning from the sale of the $i$-th commodity unit;

$x_{i}(t)$-the stocks of commodity $i$ at a time $t$;

$H$-the cost of transportation by one truck,

$h_{i}$-the purchase price per unit of $i$ commodity;

$t_{j}$-the time of truck arrival $(j=1, \ldots, k)$, the optimizable variable; 
$r_{i}^{j}$-the quantity of the $i$-th commodity at the delivery time of $t_{j}$, the optimizable variable.

Additionally we denote the quantity of sold $i$-th commodity within the time interval $\left[t_{j}, t_{j+1}\right]$ :

$$
p_{i}^{j}=\min \left(x_{i}\left(t_{j}\right), \quad \int_{t_{j}}^{t_{j+1}} \rho_{i}(t) d t\right), \quad i=1, \ldots m \quad j=1, \ldots k
$$

Where

$$
x_{i}\left(t_{j}\right)=\max \left(x_{i}\left(t_{j-1}\right)-\int_{t_{j-1}}^{t_{j}} \rho_{i}(t) d t, \quad 0\right)+r_{i}^{j}, \quad i=1, \ldots, m \quad j=1, \ldots, k
$$

Then the total flow of incoming payments from the sale of all kinds of commodities is:

$$
P=\sum_{i=1}^{m} c_{i} \sum_{j=0}^{k} p_{i}^{j}
$$

The total cost of the purchase of all types of commodities is

$$
R_{1}=\sum_{i=1}^{m} h_{i} \sum_{j=1}^{k} r_{i}^{j}
$$

The total transportation costs are

$$
R_{2}=H \cdot k
$$

We maximize the total value of cash flow, which takes into account the income from sales, as well as the purchase and delivery costs of commodities:

$$
P-R_{1}-R_{2} \rightarrow \max _{t, r}
$$

Subject to the limitations (7) on the maximum volume of cargo transported by one truck:

$$
\sum^{m} v_{i} r_{i}^{j} \leq V, \quad j=1, \ldots, k
$$

And limitations (8) for the maximum weight bf cargo transported by one truck:

$$
\sum_{i=1}^{m} m_{i} r_{i}^{j} \leq M, \quad j=1, \ldots, k
$$

Thus, the final form of the optimization model (9) can be written as follows: 


$$
\begin{gathered}
P-R_{1}-R_{2} \rightarrow \max _{t, r} \\
x_{i}\left(t_{j}\right)=\max \left(x_{i}\left(t_{j-1}\right)-\int_{t_{j-1}}^{t_{j}} \rho_{i}(t) d t, \quad 0\right)+r_{i}^{j}, \quad i=1, \ldots, m \quad j=1, \ldots, k \\
p_{i}^{j}=\min \left(x_{i}\left(t_{j}\right), \quad \int_{t_{j}}^{t_{j+1}} \rho_{i}(t) d t\right), \quad i=1, \ldots m \quad j=1, \ldots k \\
P=\sum_{i=1}^{m} c_{i} \sum_{j=0}^{k} p_{i}^{j} \\
R_{1}=\sum_{i=1}^{m} h_{i} \sum_{j=1}^{k} r_{i}^{j} \\
R_{2}=H \cdot k \\
\sum_{i=1}^{m} v_{i} r_{i}^{j} \leq V, \quad j=1, \ldots, k ; \\
\sum_{i=1}^{m} m_{i} r_{i}^{j} \leq M, \quad j=1, \ldots, k ; \\
0 \leq t_{1}, \ldots, t_{k} \leq T, \quad t_{k+1}=T ; \\
0 \leq r_{i}^{j} .
\end{gathered}
$$

For a given model, an optimal inventory control strategy $\left(t_{i}^{*}, r_{i}^{j} *\right)$ is found as the minimum of the function $P$ $R_{l}-R_{2}$ within the range $0 \leq t_{j} \leq T, 0 \leq r_{i}^{j}$ under the given limitations. This task contains $k+m * k=k(m+1)$ variables and $2 m k+3 k+3$ limitations, excluding non-negativity limitations on the variables. Substantive consideration of this problem leads to the conclusion that all deliveries can be realized simultaneously at the initial time, that is, to set $t_{j}=0, j=1, \ldots, k$. In this case, the number of problem variables will be reduced to $m$.

$$
\begin{gathered}
P-R_{1}-R_{2} \rightarrow \max _{r} \\
p_{i}=\min \left(x_{i}(0)+r_{i}, \quad \int_{0}^{T} \rho_{i}(t) d t\right), \quad i=1, \ldots, m \\
P=\sum_{i=1}^{m} c_{i} p_{i} \\
R_{1}=\sum_{i=1}^{m} h_{i} r_{i} \\
R_{2}=H \cdot k \\
\sum_{i=1}^{m} v_{i} r_{i} \leq V \\
\sum_{i=1}^{m} m_{i} r_{i} \leq M \\
0 \leq r_{i} .
\end{gathered}
$$

The Problems (9) and (10) are non-linear. Rewrite the Problem (10) in the following equivalent form (11): 


$$
\left.\begin{array}{c}
\sum_{i=1}^{m}\left(c_{i} p_{i}-h_{i} r_{i}\right)-H \cdot k \rightarrow \max _{r, p} \\
p_{i} \leq x_{i}(0)+r_{i} \\
p_{i} \leq \int_{0}^{T} \rho_{i}(t) d t=d_{i} \\
\sum_{i=1}^{m} v_{i} r_{i} \leq V, \quad \sum_{i=1}^{m} m_{i} r_{i} \leq M \\
0 \leq r_{i}, \quad 0 \leq p_{i} .
\end{array}\right\}
$$

The Problem (11) is a linear programming problem (Kremer, 2004), which can be solved, for example, by using an Excel Solver add-in. The number of arrived trucks $k$ can be taken as optimization parameter. In this case, it is sufficient to solve a series of tasks for different values of $k$ parameter, for example, using Excel SolverTable with respect to input of $k$, and choose the value of $k^{*}$, providing maximum value of functionality.

The Continuity in the problems (9)-(11), in terms of considering the variable $t$ as a continuous value, and demand, as a continuous function, plays a minimal role. In the problem (11) it is virtually non-existent, because values $d_{i}$ arecalculated separately, and in the problem (11) they are presented as deterministic numerical parameters.

The reasons to exclude the solution of the problem (9) from reducing it to the consideration of commodities supply at just the time zero, i.e. from reducing to the solution of the problem (10) and (11), may be a consideration in the model of the following factors: demand uncertainty; time value of money; the cost of commodities storage; limitations on the size of the working capital, the capacity of storage space, the size (number) of the trucks, the limitations on shelf life, and so on.

Inaccuracy of distant forecasts inspires to move the supply point closer to the point of sale. However, there is a reverse trend: an early delivery of the commodities insures against the lack of commodities at the extraordinarily increased demand. As an example of this type of models, we consider a model that takes into account the time value of money.

2.2 Multivariate Models to Determine Simultaneously Optimal Supply Points and the Delivery Scopes with Due Consideration of the Time Value of Money

From the standpoint of such disciplines, as financial analysis, financial management, and financial mathematics, there is a need to improve the optimizing methods of inventory management strategies to incorporate the factor of the time value of money and maximize the effectiveness of the similar logistics systems (Brodetsky, 2007). It is exactly a direct integration of the time value of money that has essential effect on the parameters of the optimal inventory control strategy (Brigham, 2011).

The problem of optimization of diversified inventory management system is described in a study for the case of payment for procurement and transportation on a "prenumerando" basis, which corresponds to the implementation of such payments at a time of total delivery of the shipment at the beginning of the period between deliveries. For simplicity, incoming payments from the sale of commodities are also correlated with the initial point of the relevant period.

Given the above described, further we introduce the following notation:

$\mu$-the coefficient of the time-dependent value of money, which provides a multiplicative increase in the cost of money by a factor of $\exp (\mu)$ per unit time,

$t_{j}^{i *}$-the point in time corresponding to the end of commodity $r_{i}^{j}$ after the arrival of the truck at a time $t_{j}$, which is determined from the integral Equation (12):

$$
x_{i}\left(t_{j}\right)=\int_{t_{j}}^{t_{j}^{i *}} \rho_{i}(t) d t, \quad i=1, \ldots, m, \quad j=0, \ldots, k
$$


Next, introduce the value $\tau_{i}^{j}=\min \left(t_{j+1}, t_{j}^{i} *\right), \quad i=1, \ldots, m, \quad j=0, \ldots, k$ which will allow us to determine the period of the actual consumption of commodities within the time interval between deliveries $\left[t_{j}, t_{j+1}\right]$. Graph that represents changes in the inventory level is shown in Figure 1.

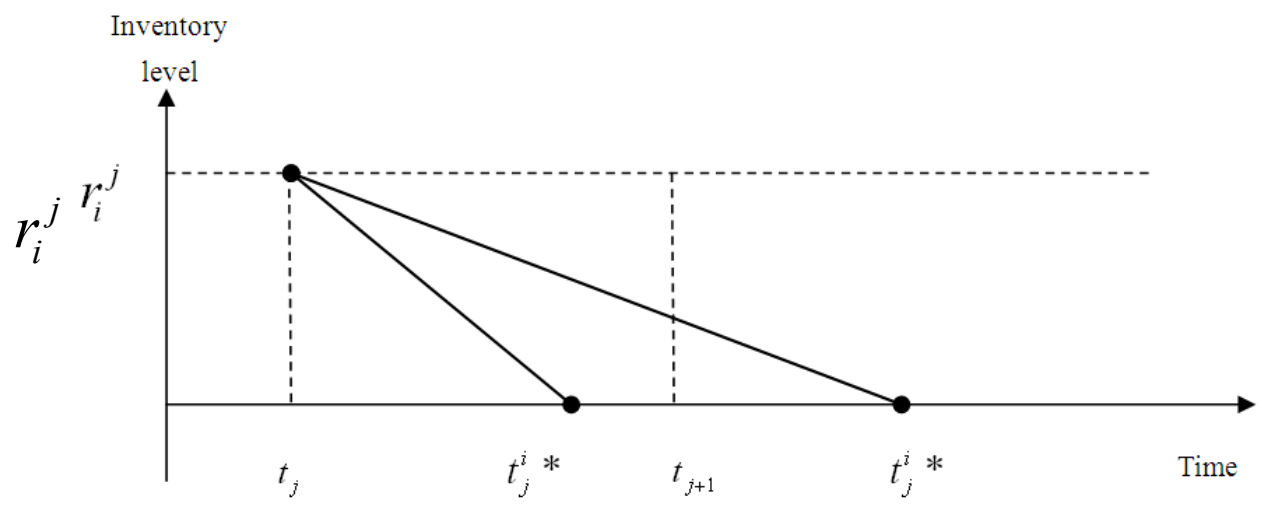

Figure 1.Changes in inventory level

Taking into account the notation introduced earlier, we obtain discounted cash flows of incoming payments from the sale of commodities and cost-intensive flows of payments for the commodities procurement and transportation expenses:

- from the sale of commodities:

$$
P^{k}=\sum_{i=1}^{m} c_{i} \sum_{j=0}^{k} \int_{t_{j}}^{\tau_{j}^{j}} \frac{\rho_{i}(t)}{e^{\mu t t_{j}}} d t
$$

- cost-intensive flow of payments for procurement of commodities:

$$
R_{1}^{k}=\sum_{i=1}^{m} h_{i} \sum_{j=1}^{k} \frac{r_{i}^{j}}{e^{\mu t_{j}}}
$$

- cost-intensive flow of payments for transportation expenses:

$$
R_{2}^{k}=H \cdot \sum_{j=1}^{k} \frac{1}{e^{\mu t_{j}}}
$$

Then the inventory management optimization model (16), taking into account the time value of money, is as follows: 


$$
\begin{gathered}
P^{k}-R_{1}^{k}-R_{2}^{k} \rightarrow \max _{t, r} \\
x_{i}\left(t_{j+1}\right)=x_{i}\left(t_{j}\right)-\min \left(x_{i}\left(t_{j}\right), \int_{t_{j}}^{t_{j+1}} \rho_{i}(t) d t\right)+r_{i}^{j+1}, \quad j=0, \ldots, k \quad i=1, \ldots, m \\
x_{i}\left(t_{j}\right)=\int_{t_{j}}^{t_{j}^{i *}} \rho_{i}(t) d t, \quad i=1, \ldots, m, \quad j=0, \ldots, k \\
\tau_{i}^{j}=\min \left(t_{j+1}, t_{j}^{i} *\right), \quad i=1, \ldots, m, \quad j=0, \ldots, k \\
P^{k}=\sum_{i=1}^{m} c_{i} \sum_{j=0}^{k} \int_{t_{j}}^{\tau_{j}^{j}} \frac{\rho_{i}(t)}{e^{\mu t_{j}}} d t \\
R_{1}^{k}=\sum_{i=1}^{m} h_{i} \sum_{j=1}^{k} \frac{r_{i}^{j}}{e^{\mu t t_{j}}} \\
R_{2}^{k}=H \cdot \sum_{j=1}^{k} \frac{1}{e^{\mu t_{j}}} \\
\sum_{i=1}^{m} v_{i} r_{i}^{j} \leq V, \quad j=1, \ldots, k \\
\sum_{i=1}^{m} m_{i} r_{i}^{j} \leq M, \quad j=1, \ldots, k \\
T_{\min } \leq t_{1}, \ldots, t_{k} \leq T \\
T_{k+1}=T \\
0 \leq r_{i}^{j} .
\end{gathered}
$$

As noted earlier, the inventory management models for specific organizations and enterprises differ essentially depending on a combination of factors that are taken into account. Next, we present various modifications of the diversified inventory management model, taking into account the time value of money, depending on the factors and limitations considered.

1) Accounting for the limitations on the capital size

There are circumstances, where an enterprise is limited in the free resources or has no ability to use borrowed funds.

For mathematical formalization of this condition, we introduce the following notation:

$S_{0}-$ is the amount of available funds in time point $t=t_{0}, \mathrm{~s}-$ is the index of period, $s=1, \ldots, k$.

The discontinuity condition in the cash flow is represented in the form of limitation (17):

$$
S_{0}+P^{s}-R_{1}^{s}-R_{2}^{s} \geq 0, \quad s=1, \ldots, k .
$$

2) Accounting for the limitations on the batch quantity

Often supplying companies carry out the delivers of the commodities in the amount not less than the fixed volume; or consignment of certain volume is sold at a substantial discount or at a special price (the policy of flexible prices). In this case, for example, there is a limit on the minimum amount of consignment (18), which can be represented as follows: 


$$
R_{i}^{\min } \leq r_{i}^{j}
$$

However, on the other hand, stocks of commodities at supplier companies may also be limited, at that; this limitation may have a dynamic nature. With this circumstance in mind, the limitation (20) would be more complete if presented in the following form:

$$
R_{i}^{\min } \leq r_{i}^{j} \leq R_{i}\left(t_{j}\right),
$$

where $R_{i}\left(t_{j}\right)$-is the maximum available quantity of commodity $i$ in a stock at the time point $t_{j}$.

3) Accounting for different types of transport means

If in the inventory management system described, at the company's disposal there are several ways to deliver the commodities, for example, several types of trucks, and one has to select among them the most optimum truck, then we denote:

$n$-the number of truck types $l=1, \ldots, n$,

$V_{l}, M_{l}, H_{l}$-the maximum amount of cargo, maximum cargo weight and the cost of transportation per one truck of $l$ type,

$L=\left(l_{l}, \ldots, l_{k}\right)$-number of trucks types, engaged in the $k$-th delivery $1 \leq l_{i} \leq n, \quad l_{i} \in N$.

In this case, it is logical to consider the discrete elements of the $L$ vector as the optimization parameters.

4) Accounting for the temporal dynamics of the system parameters

In some cases, to improve the adequacy and accuracy of the models, it is appropriate to consider changes over time of some characteristics of the commodities delivery and sale process. In this case, time dependence functions are considered instead of relevant fixed numerical parameters. Typically, these functions are the result of the market forecast models. In particular, in case the commodities value is changed, we denote the purchase price per unit of good $i$ in a time point $t_{j}$ as $h_{i}\left(t_{j}\right)$.

Similarly, the revenues from sales of commodity unit $i$ within the time interval between deliveries $\left[t_{j}, t_{j+l}\right]$ can be expressed by the function $c_{i}\left(t_{j}\right)$.

In case the cost of transportation changes, we denote the transportation cost by the truck at the time point $t_{i}$ as $H\left(t_{j}\right)$. In this case, our inventory control optimization model takes the form of (20): 


$$
\begin{aligned}
& P^{k}-R_{1}^{k}-R_{2}^{k} \rightarrow \max _{t, r, L} \\
& x_{i}\left(t_{j+1}\right)=x_{i}\left(t_{j}\right)-\min \left(x_{i}\left(t_{j}\right), \int_{t_{j}}^{t_{j+1}} \rho_{i}(t) d t\right)+r_{i}^{j+1}, \quad j=0, \ldots, k \quad i=1, \ldots, m \\
& x_{i}\left(t_{j}\right)=\int_{t_{j}}^{t_{j}^{* * *}} \rho_{i}(t) d t, \quad i=1, \ldots, m, \quad j=0, \ldots, k \\
& \tau_{i}^{j}=\min \left(t_{j+1}, t_{j}^{i} *\right), \quad i=1, \ldots, m, \quad j=0, \ldots, k \\
& P^{s}=\sum_{i=1}^{m} \sum_{j=0}^{s} \int_{t_{j}}^{\tau_{j}^{\prime}} \frac{c_{i}\left(t_{j}\right) \rho_{i}(t)}{e^{\mu t_{j}}} d t, \quad s=1, \ldots, k \\
& R_{1}^{S}=\sum_{i=1}^{m} \sum_{j=1}^{s} \frac{h_{i}\left(t_{j}\right) r_{i}^{j}}{e^{\mu t_{j}}}, \quad s=1, \ldots, k \\
& R_{2}^{S}=\sum_{j=1}^{s} \sum_{l=1}^{n} \frac{H_{l}\left(t_{j}\right)}{e^{\mu t_{j}}}, \quad s=1, \ldots, k \\
& S_{0}+P^{s}-R_{1}^{s}-R_{2}^{s} \geq 0, \quad s=1, \ldots, k \\
& \sum_{i=1}^{m} v_{i} r_{i}^{j} \leq V_{l_{k}}, \quad j=1, \ldots, k \\
& \sum_{i=1}^{m} m_{i} r_{i}^{j} \leq M_{l_{k}}, \quad j=1, \ldots, k \\
& R_{i}^{\min } \leq r_{i}^{j} \leq R_{i}\left(t_{j}\right), \quad i=1, \ldots, m \\
& T_{\min } \leq t_{1}, \ldots, t_{k} \leq T \\
& T_{k+1}=T, 0 \leq r_{i}^{j} .
\end{aligned}
$$

Often the purchased batch size is the determining factor in the formation of commodity price. Therefore, accounting for the flexible price formation policy is an important condition, when modeling the optimal inventory control strategy.

We denote the purchase price per unit of commodity $i$ at a time point $t_{j}$, depending on the batch purchase size as $h_{i}\left(t_{j}, r_{i}^{j}\right)$.

Then the integral costs of commodities procurement, taking into account the time value of money, will be as follows:

$$
R_{1}^{s}=\sum_{i=1}^{m} \sum_{j=1}^{s} \frac{h_{i}\left(t_{j}, r_{i}^{j}\right) \cdot r_{i}^{j}}{e^{\mu t_{j}}}
$$

2.3 Model to Determine Simultaneously the Optimal Time of Delivery and the Scope of Supply in a Single-Commodity Case

Obtaining analytical solution of the model (16) is quite difficult, thus consider the special case of the model (16), namely single-commodity model (22). 


$$
\begin{gathered}
P^{k}-R_{1}^{k}-R_{2}^{k} \rightarrow \max _{t, r} \\
x\left(t_{j+1}\right)=x\left(t_{j}\right)-\min \left(x\left(t_{j}\right), \int_{t_{j}}^{t_{j+1}} \rho(t) d t\right)+r_{j+1} \\
x\left(t_{j}\right)=\int_{t_{j}}^{t_{t}^{*}} \rho(t) d t \\
\tau_{j}=\min \left(t_{j+1}, t_{j} *\right) \\
P^{k}=c \cdot \sum_{j=0}^{k} \int_{t_{j}}^{\tau_{j}} \frac{\rho(t)}{e^{\mu t}} d t \\
R_{1}^{k}=h \cdot \sum_{j=1}^{k} \frac{r_{j}}{e^{j t_{j}}} \\
R_{2}^{k}=H \cdot \sum_{j=1}^{k} \frac{1}{e^{\mu t_{j}}} \\
v \cdot r_{j} \leq V \\
m \cdot r_{j} \leq M \\
T_{\min } \leq t_{1} \leq t_{2} \leq \ldots \leq t_{k} \leq T_{\max } \\
0 \leq r_{j} .
\end{gathered}
$$

Obviously, in the case of a single-commodity, delivery of regular batch of commodities takes place only after the disposal of the previous one. Therefore, the structure of the optimal solution will have the form, shown in Figure 2. As a consequence, the problem admits more simple representation (23).

Let introduce the following notation:

$r_{i}$-the quantity of commodity in the $i$-th truck;

$t_{i}$-the arrival time of $i$-th truck;

$\tau_{i}\left(t_{i}, r_{i}\right)$-the time of commodity disposal in the amount of $r_{i}$ starting from the time $t_{i}$.

$$
\begin{gathered}
P^{k}-R_{1}^{k}-R_{2}^{k} \rightarrow \max _{t, r} \\
\int_{t_{i}}^{\tau\left(t_{t}, r_{i}\right)} \rho(t) d t=r_{i}, \quad i=1, \ldots, k \\
P^{k}=c \cdot \sum_{j=0}^{k} \int_{t_{j}}^{\tau_{j}\left(t, r_{j}\right)} \frac{\rho(t)}{e^{\mu t}} d t \\
R_{1}^{k}=h \cdot \sum_{j=1}^{k} \frac{r_{j}}{e^{\mu t_{j}}}, \\
R_{2}^{k}=H \cdot \sum_{j=1}^{k} \frac{1}{e^{\mu t_{j}}}, \\
v \cdot r_{i} \leq V, \quad i=1, \ldots, k, \\
m \cdot r_{i} \leq M, \quad i=1, \ldots, k .
\end{gathered}
$$




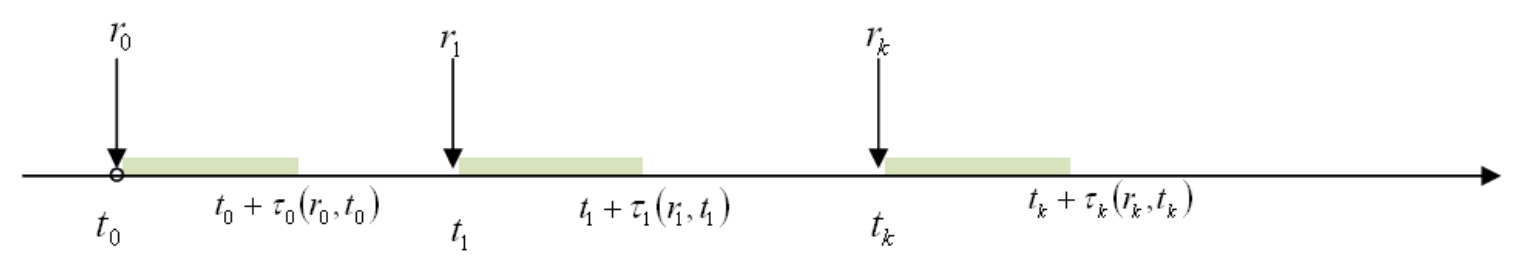

Figure 2. The structure of optimal supply for single-commodity case

It is logical to assume that in the absence of any specific limitations, truck is always ordered in the shortest possible time $T_{\min }$ before delivery, because at this point the enterprise has more reliable forecasts on demand for commodities than in the case of earlier orders. In this context, although the optimization problem solution considers several deliveries in different periods, the goal of solving this optimization problem is to find the optimal values of the parameters only for the first delivery, namely $t_{1}^{*}$ and $r_{1}^{*}$.

It is further proposed to use the following rule: if $t_{1}{ }^{*}>T_{\min }$ the order is not placed. If $\mathrm{t}_{1}{ }^{*}=T_{\min }$, then the order is made in a quantity of $\mathrm{r}_{1}{ }^{*}$.

Therefore, it seems appropriate to consider the problem of optimal delivery by just one truck.

At that, the optimal solution may turn out to be non-trivial to the extent that:

- Delivery by truck is carried out not by the time of the commodity completion;

- Delivery by truck is carried out not in full volume.

Delivery diagrams for the trivial and nontrivial cases are shown in Figures 3 and 4, respectively.

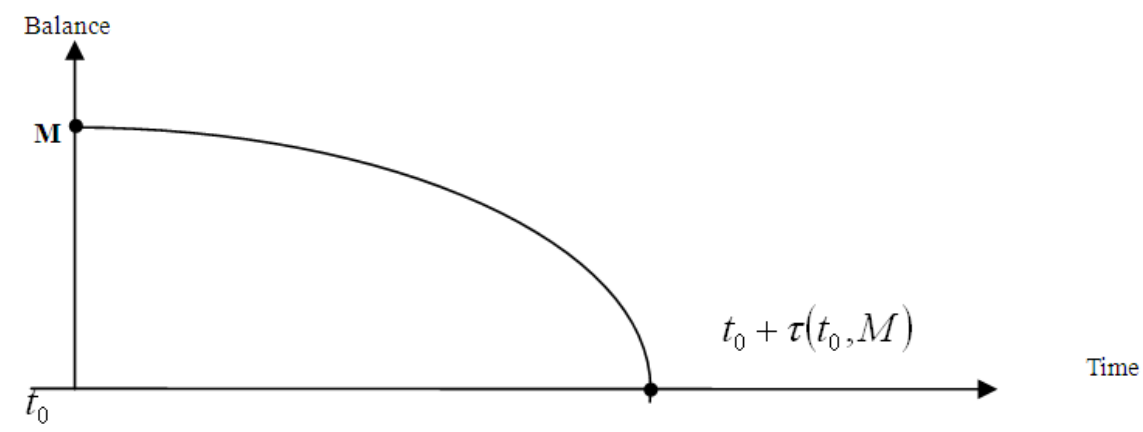

Figure 3. Delivery of full truck by the time of commodity completion (trivial solution)

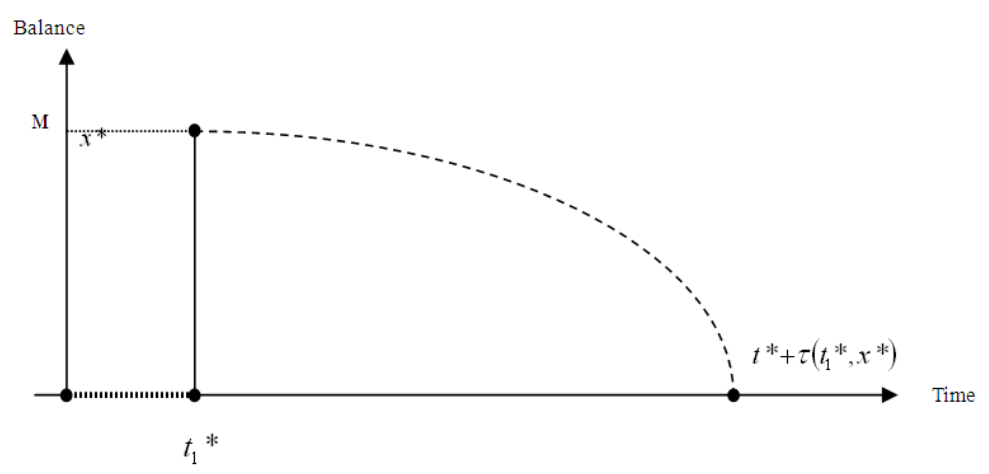

Figure 4. Delayed delivery of partly-loaded truck (non-trivial solution)

Let's carry out an analysis of the model to determine the quantity of commodities and delivery time points for 
the case of one truck and one product.

Figures 5 and 6 schematically show time points pattern corresponding to the delivery and completion of commodities.

The mathematical formulation of the problem (24) and (25) in this case can be written as:

$$
\left.\begin{array}{c}
F(t, x)=c \int_{t}^{t+\tau(t, x)} \frac{\rho(t)}{e^{\mu t}} d t-\frac{h x+H}{e^{\mu t}} \rightarrow \max _{t, x} \\
\int_{t}^{t+\tau(t, x)} \rho(t) d t=x \\
t \leq T \\
x \leq M \\
x \geq 0 \\
t \geq 0
\end{array}\right\}
$$

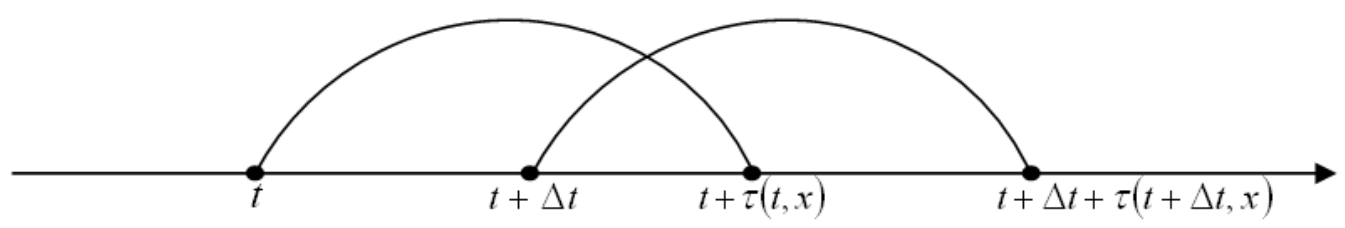

Figure 5. Time points pattern corresponding to commodity delivery and completion

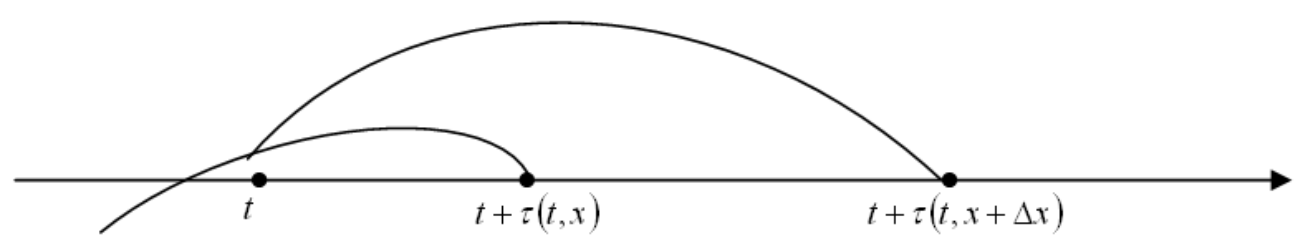

Figure 6. Time points pattern corresponding to commodity delivery and completion

As a result of mathematical transformations, we obtain a system of nonlinear equations to find the optimal values of $t^{*}$ and $x^{*}$,i.e. delivery time point and size parameters. The solution to this system can be obtained using numerical methods for finding equation roots (Vasilyev, 2002).

\subsection{A Stochastic Simulation Model}

Another research area is related to the development and implementation into practice of simulation and optimization approach to the organization of the inventory management process under uncertainty (Sviridova \& Kosorukov, 2013).

The question on how much commodities and when should be ordered to reach the optimal business performance operation, taking into account technological, financial and resource limitations and variability of market environment, still requires the answer. The Optimization and simulation methods, as well as their combination allow one to solve the problem and build a business strategy of inventory management (Mitsel, 2005).

The author has developed a set of inventory management models applicable under conditions of uncertainty, including simulation and optimization model for determining the two parameters of the strategy, as well as the dynamic modification of the proposed model.

The models make it possible:

- To find the optimal size of shipments and the best time for their delivery; 
- To take into account the uncertainty of demand and the time of delivery;

- To select in the simulation modeling the optimum combination of partners for trading company, namely suppliers, transport company and credit institution, depending on their operation environment.

- The features and assumptions of simulation model include:

- The model describes the activities of the trading company in the field of inventory management for a certain time line, consisting $n$ periods of a given duration;

- A few suppliers and transport companies are potential partners of the trading company; several options of credit products are available;

- Specifics of the business is such that the decision-maker has to develop a delivery schedule for the concerned time period and to determine the optimal combination of business partners, i.e. To identify a supplier to place the order, a transport company to deliver shipments, as well as to choose the most suitable credit product;

- Company sells several kinds of commodities; each commodity is characterized by a set of parameters (sales price, cost of storage, and the purchase price), known for each period;

- There is uncertainty with respect to the demand for commodities, as well as delivery time;

- The simulation model determines also optimum scope of order for each type of commodity in each time period, as well as the optimal combination of partners and the credits available, for which the total expected cost of the project will be minimal;

- Total costs of the company are presented as a function of (26):

$$
I=I_{1}+I_{2}+I_{3}+I_{4}+I_{5} .
$$

The cost of storing stock $I_{l}$ reflects the costs of stock maintenance in the warehouse and includes the cost of storage, maintenance and care.

Losses from the shortage of commodities $I_{2}$ include the potential losses of revenue due to lack of stock, subject to the availability of demand.

The Transportation costs $I_{3}$.

The credit servicing costs $I_{4}$ include accrued interest on the credit.

The costs of purchasing order $I_{5}$ include expenses associated with placing an order with the supplier.

Let us describe some additional assumptions of the model:

- The model allows using only short-term credits for just one period. In this type of landing one may take money in the beginning of the first period, and return money at the end of the period. Interest is payable at the rate of a given period calculated by the simple interest formula (27):

$$
A_{i}=D_{i}\left(1+r_{i}\right)
$$

where $D_{i}$-is the amount of a credit in the period $i, A_{i}$-is the refund amount in the period $i, r_{i}$-is the interest rate on the credit in the period $i$.

The Models of this type of credit can include, for example, credit lines, overdrafts, and commercial short-term crediting.

In this model, we consider a credit line with a disbursement limit, i.e., the total amount of funds granted to the borrower under the credit line does not exceed the maximum limit in amount of $D^{\prime \prime}$; there is also a condition for a minimum amount of the credit $D^{\prime}$.

- If the company does not return credit at the end of the period, the amount of debt is carried over to the next period and the interest is calculated in the same way without penalty.

- The cost for storage is taken as average over a concerned period and depends only on the quantity of commodities in stock and storage cost during the period.

Input data: 
$c_{i j}$-the price of good $j$ in the period $i$,

$z_{i j}$-the cost of storing commodity $j$ in the period $i$,

$S_{i j}$-the purchase price of commodity $j$ in the period $i$,

$F_{i j}$-the minimum order quantity from the supplier,

$p_{i}$-the cost of delivery by the transport company in the period $i$,

$r_{i}$-the interest rate on credit in the period $i$,

$t_{i}$-the duration of the period $i$,

$L_{0 j}$-the initial inventories of the commodity $j$

$\Delta_{0}$-seed money,

$D^{\prime}$-the minimum amount of credit,

$D^{\prime \prime}$-the maximum amount of credit.

We also denote the following:

$d_{i j}$-the demand for commodity $j$ in the period $i$, which is a random variable;

$\tau_{i}$-the delivery time of commodities by transport company, ordered in the period $i$, which is a random variable;

We need to find:

$x_{i j}$-the quantity of ordered commodity $j$ in the period $I$.

The optimization criterion is a minimum of total expected costs.

Let denote additionally:

$D_{i}$-the amount of credit in the period $i$,

$Q_{i j}$-the number of delivered commodities $j$ in the period $i$,

$$
Q_{i j}=\left\{\begin{array}{ccc}
x_{i-1, j}, \quad \tau_{i-1} \geq t_{i-1}, \quad \tau_{i} \geq t_{i} \\
x_{i-1, j}+x_{i j}, \quad \tau_{i-1} \geq t_{i-1}, \quad \tau_{i}<t_{i} \\
x_{i j}, \quad \tau_{i-1}<t_{i-1}, \quad \tau_{i}<t_{i}
\end{array}\right.
$$

$M_{i j}$-the number of commodities $j$ in stock at the beginning of the period $I$; 


$$
M_{i j}=Q_{i j}+L_{i-1, j},
$$

$K_{i j}$-the amount of commodities $j$ outsold in the period $I$;

$$
K_{i j}=\min \left(d_{i j}, M_{i j}\right),
$$

$L_{i j}$-the balance of commodity $j$ at the end of the period $I$;

$$
L_{i j}=M_{i j}-K_{i j}
$$

$d_{i j}^{\prime}$-backlog demand;

$$
d_{i j}^{\prime}=d_{i j}-K_{i j} .
$$

The movement of stocks in the period is shown in Figure 7:

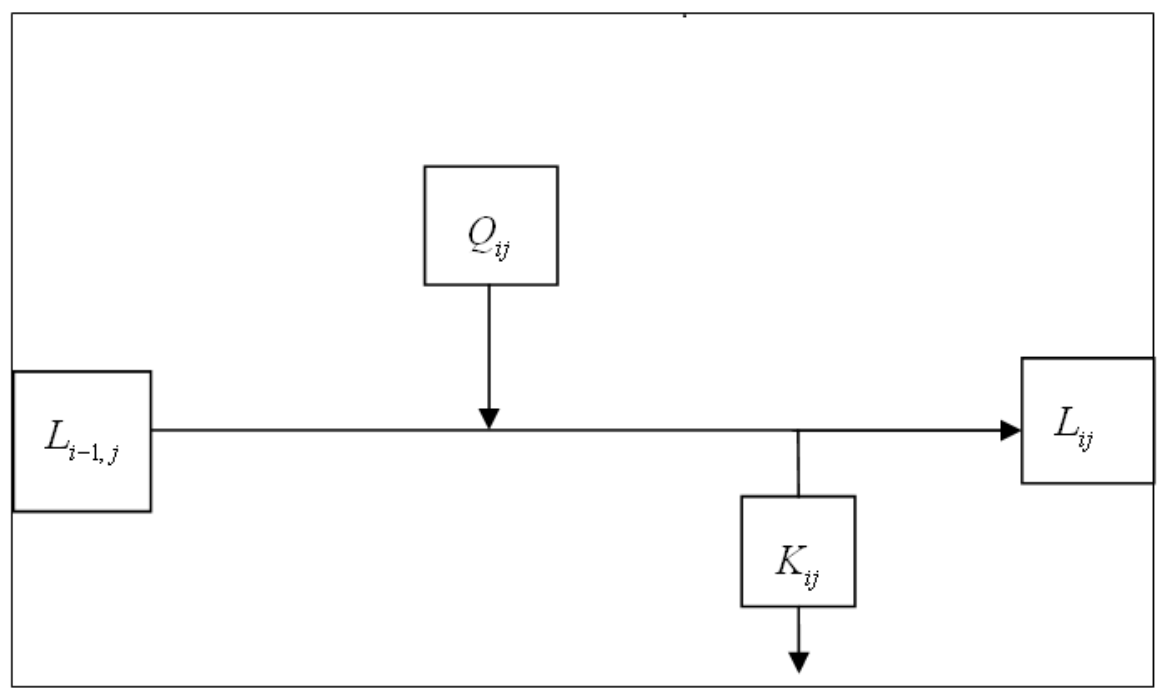

Figure 7. Schematic representation of the change in the stock of commodity $j$ over the period $i$

Let us further denote:

$P_{i j}$-the revenue from sale of commodity in the period $i$,

$$
\begin{gathered}
P_{i j}=K_{i j} \cdot c_{i j}, \quad P_{i}=\sum_{j} P_{i j}, \\
D_{i}=\max \left\{0 ; \sum_{j} x_{i j} \cdot s_{i j}-\Delta_{i-1}\right\}-\text { the amount of a credit in the period } i
\end{gathered}
$$

$\Delta_{i}$-cash balance at the end of the period $i$

$$
\Delta_{i}=\Delta_{i-1}+D_{i}-\sum_{j} x_{i j} \cdot s_{i j}-\sum_{j} p_{i} \cdot x_{i j}-\sum_{j} L_{i j} \cdot z_{i j}+\sum_{j} P_{i j}-D_{i} \cdot\left(1+r_{i}\right)
$$

The Costs of storage for all commodities in the period $i$ 


$$
I_{1 i}=\sum_{j} L_{i j} \cdot z_{i j}
$$

The Costs associated with a deficit for all commodities in the period $i$

$$
I_{2 i}=\sum_{j} d_{i j}^{\prime} \cdot c_{i j}
$$

The Transportation costs in the period $i$

$$
I_{3 i}=\sum_{j} p_{i} \cdot x_{i j} .
$$

The costs associated with credit repayments in the period $i$

$$
I_{4 i}=\left(D_{i} \cdot r_{i}\right)
$$

The Order placement costs in the period $i$

$$
I_{5 i}=\sum_{j} x_{i j} \cdot S_{i j}
$$

Cash flows for each period are presented schematically in Figure 8:

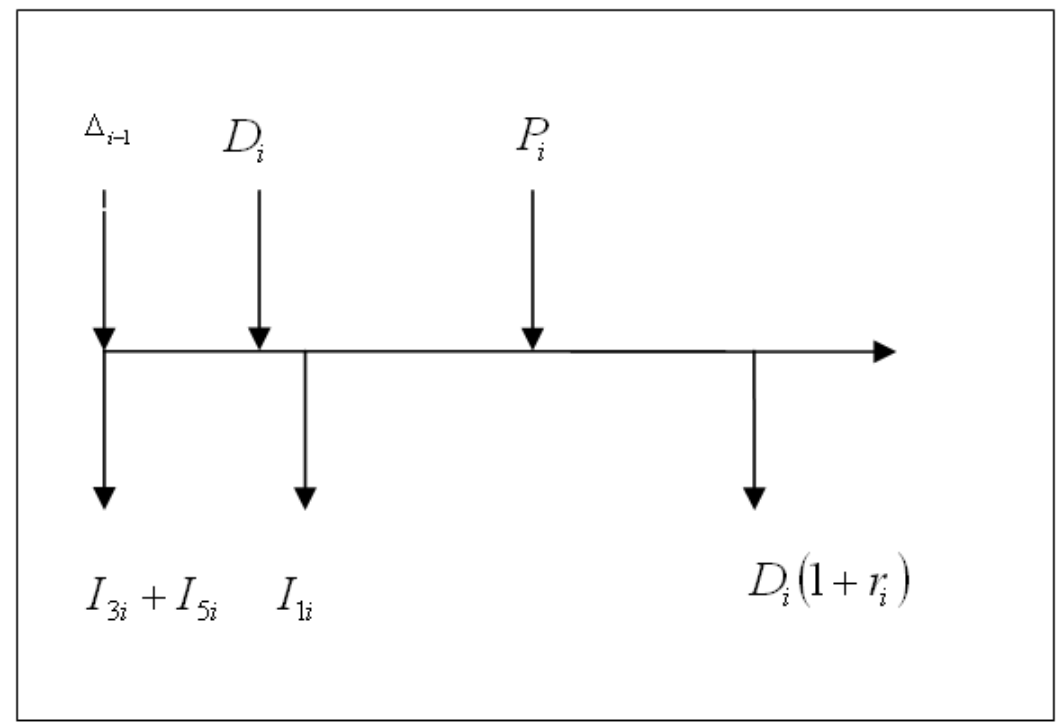

Figure 8. Conditional presentation of cash flows in the period $i$

Then, taking into account the notations, we can write the costs as follows:

Storage costs

$$
I_{1}(x)=\sum_{i} I_{1 i}=\sum_{i} \sum_{j} L_{i j} \cdot z_{i j}
$$

We can write similarly: the costs of the deficit, the transportation costs, the credit costs, and the costs of ordering: $I_{2}(x), I_{3}(x), I_{4}(x), I_{5}(x)$

The total costs:

$$
I(x)=I_{1}(x)+I_{2}(x)+I_{3}(x)+I_{4}(x)+I_{5}(x)
$$

Note that, since $\tau_{i}, d_{i j}$ are random variables, then the values of $Q_{i j}, M_{i j}$, depending on them, are the random values as well, though their distribution laws are not derived analytically. 
In this case the optimization criterion is the minimum of total expected costs. Thus, we turn to the average expected costs:

Let us generate in our simulation model, for example, 1000 random values of demand for each type of commodity and the time of delivery. Then the average cost can be calculated as:

$$
\bar{I}_{1}(x)=\frac{I_{1}^{1}(x)+\ldots+I_{1}^{1000}(x)}{1000}
$$

The values $\bar{I}_{2}(x), \quad \bar{I}_{3}(x), \quad \bar{I}_{4}(x), \quad \bar{I}_{5}(x)$ are calculated similarly.

$$
\bar{I}(x)=\bar{I}_{1}(x)+\bar{I}_{2}(x)+\bar{I}_{3}(x)+\bar{I}_{4}(x)+\bar{I}_{5}(x)
$$

Thus, the stochastic simulation and optimization model to minimize the costs of inventory management will be as follows:

\section{Results and Discussion}

$$
\begin{gathered}
\bar{I}(x) \rightarrow \min _{x} \\
x_{i j} \geq F_{i j} \\
D^{\prime} \leq D_{i} \leq D^{\prime \prime} \\
x_{i j} \geq 0, \quad i=1,2, \ldots n ; \quad j=1,2, \ldots m
\end{gathered}
$$

The Figure 9 shows an iterative algorithm that describes the operation of the model and allows for calculating the optimal parameters of inventory control strategy in the selected software environment.

For practical solution of this problem one can take advantage, for example, of RISK Optimizer optimization add-in for Microsoft Excel, which combines simulation modeling technologies @RISK (Microsoft Excel add-in for risk analysis of Palisade company) with a genetic optimization algorithm that allows to build the optimization models, which include uncertainty of the different nature both in composite function and limitations (Brodetsky, 2012).

When testing the model, the author obtained the results, which indicated that compared with an approach based on the average projected values of demand, the use of approaches based on the optimization and simulation modeling to form the stocks with allowances made for the uncertainties in the parameters of inventory control strategy, can reduce costs by an average of $20 \%$.

An important issue of a trading company is the choice of partners, i.e. suppliers, transportation companies, and credit institutions. The characteristics of the companies, operating and offering their services in the market nowadays, are quite contradictory. In such a variety of options, it is often impossible to make a choice analytically, whereas the best choice for partners may bring additional revenue opportunities and reduce costs. There are traditional methods for evaluation and selection of partners, such as, for example, point-based system or analytic hierarchy process (Saaty, 1993). The problem concerns the rational choice of methodology, since each method has its drawbacks, such as the subjective assessment, consideration of a large number of criteria to assess the extensive, not always unambiguous information, and others.

The advantage of the simulation and optimization approach is the ability to model the company's operations in different conditions, to see the result in each case and make a quantitively informed choice to the benefit of the best combination of partner companies.

Thus, the simulation modeling, which allows to take into consideration a huge number of parameters that are of the random nature and have complex cause-and-effect relationships (transportation tariffs, limitations on the use of different types of delivery and trucks, and others), seems to be the only tool for solving stocks optimization problems and other issues related to the organization of the logistics process (Emelyanov, 2002).

However, according to the logistics experts, in contrast to industrialized countries, the countries of the former USSR just start using the simulation modeling, while simulation modeling technologies are employed only by a few large companies. In the majority of commercial enterprises, management decisions on the organization of 
commodity flows are taken with a focus on the average indicators of demand and delivery time that reduces the effectiveness and efficiency of trading activity, increasing the costs of storage and lost profits (Sergeev, 2001).

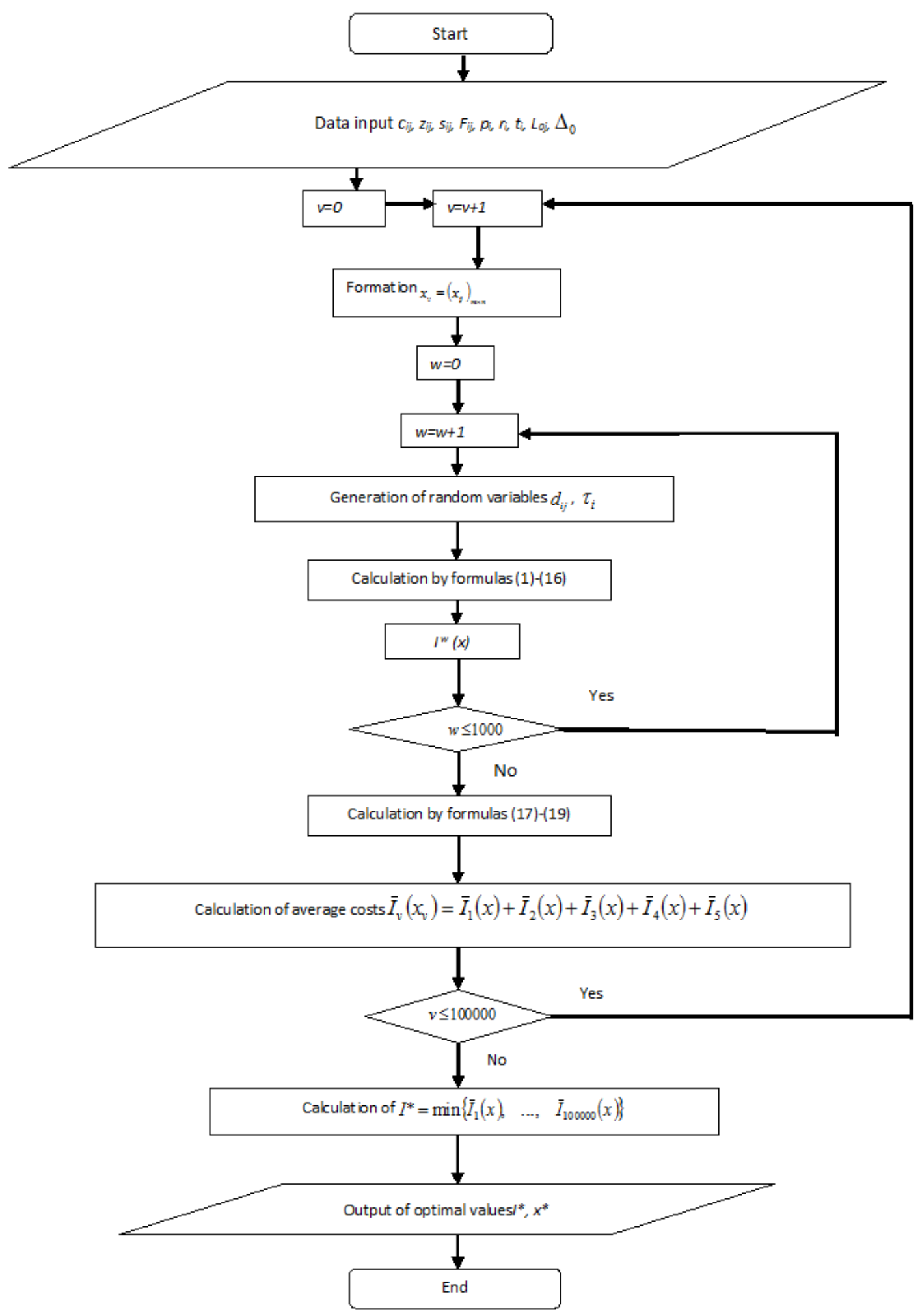

Figure 9. Model operation algorithm 
A promising area for further research would be the development of a dynamic model for supply planning, the essence of which is as follows.

As before, there is a certain time horizon of forecasting the demand for certain types of commodities. Unlike the case considered above, there is no need to form immediately a delivery schedule for the entire period. Since there is a minimum time of the order $T_{\min }$, the commodities cannot be delivered earlier than the time of order. In this case, it is obvious that placing the order at the time point $T>T_{\min }$ is irrational, because it increases the uncertainty and certainly is not optimal. For this reason, the problem whether or not placing the order at the time point $t+T_{\min }$ is reasonable is solved at each point of time $t$ (e.g., day) (Sviridova, 2014).

Figure 10 presents some heuristic algorithm based on the simulation and optimization model, shown above, which describes the dynamic process of decision-making procedure at each time $t$.

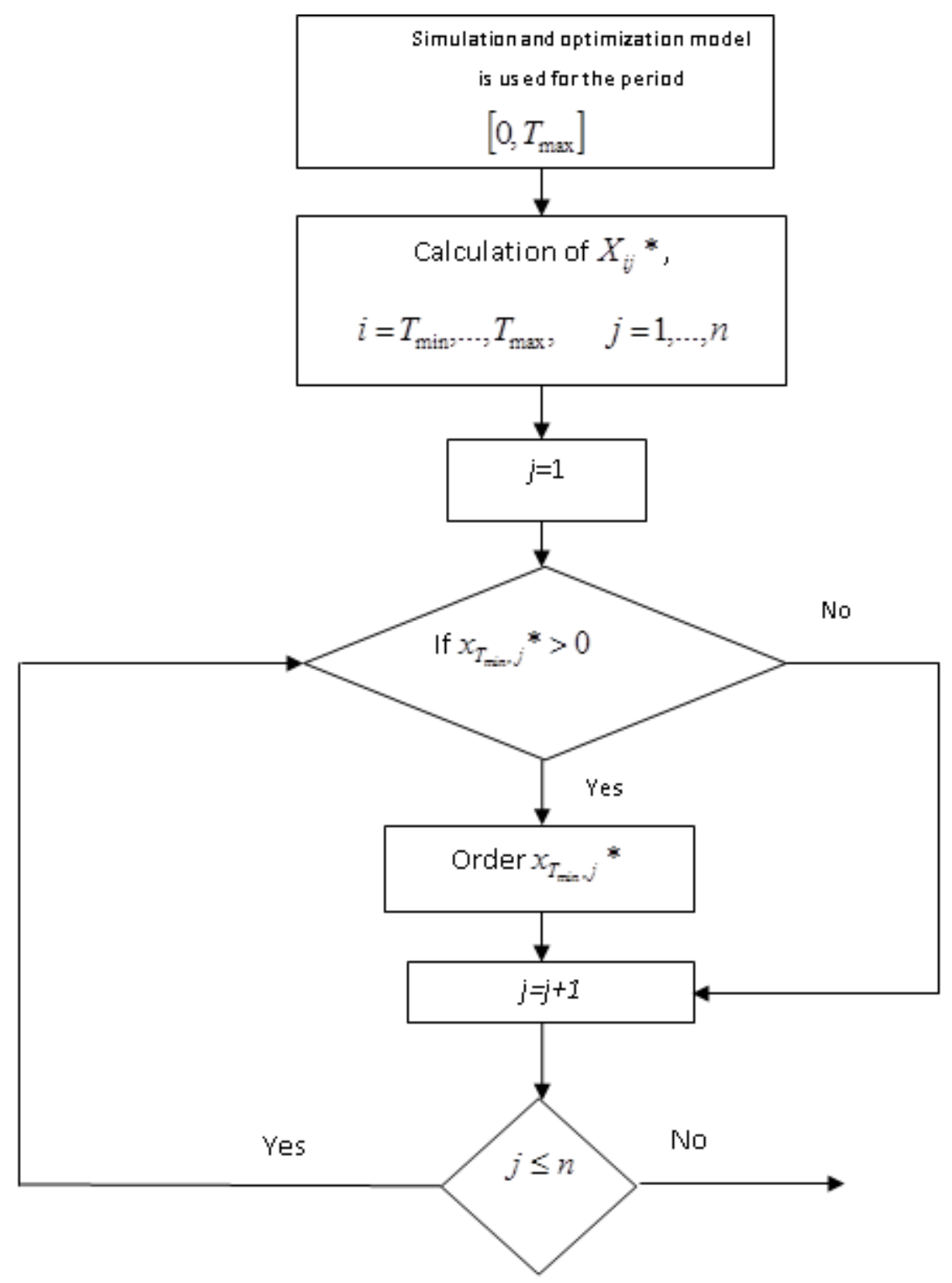

Figure 10.The diagram of algorithm for dynamic scheduling of deliveries

This model is used at any given time $t$ (e.g., daily) after updating the input information.

Note also that the model is functioning correctly in the absence of uncertainty on demand or delivery time, as 
well as in case of the presence of just one kind of uncertainty.

This model also allows not only optimizing the delivery process throughout its duration, but carrying the optimal choice of partners: suppliers of commodities, transportation company, and credit institution. In this case one has to conduct experiments on certain scenarios, desirably within the typical time periods of company operation for the various combinations of counterparties, using their parameters as input data to the model.

\section{Conclusion}

The Simulation approach allows playing scenarios of real processes and situations when defining the strategy and decision-making rules with respect to management of commercial organizations, which in turn provides the basis for informed choice of actions under the conditions of incomplete information about the future.

Having constructed the simulation and optimization model, which calculates the optimal order parameters for various combinations of suppliers, transport companies and credit institutions (i.e., calculating the model with different input data relevant to the counterparties operation environment), we can find the optimal combination of contractors for the company operation at minimum cost within the concerned time period.

It can be shown that the use of the simulation and optimization approach together with the developed models make it possible to improve the integrated financial results of the operating company for a certain period by $20 \%$ (as compared with a result that gives the ordering strategy based on the average projections of demand). Thus, simulation modeling is a versatile tool for both choosing the optimal long-term strategy for inventory management and operational decision-making with respect to the commodity flow organization.

\section{References}

Brigham, J. (2011). Financial management. Express course (4th ed.). Houston.

Brodetsky, G. (2007). Inventory management. Textbook. Moscow: Eksmo.

Brodetsky, G. (2012). Economic-mathematical methods and models in logistics. Optimization procedures. Moscow: Academy Publishing Center.

Emelyanov, A. (2002). Simulation modeling of economic processes: Textbook. Moscow: Finance and Statistics.

Goldsbi, T. (2009). Lean manufacturing and six sigma in logistics. Manual on logistic processesoptimization. Moscow: Grevtsov Pablisher.

Kosorukov, O. (2005). Methods for quantitative analysis in business. Textbook. Moscow: INFRA-M.

Kremer, N. (2004). Operations research in economics. Moscow: UNITY.

Lukinskiy, V. (2007). Models and methods of the logistics theory. Textbook (2nd ed.).St.-Petersburg: Peter.

Mitsel, A. (2005). Simulation modeling of economic objects. Laboratory course. Tomsk: NTL Publishing House.

Prosvetov, G. (2008). Mathematical methods in logistics. Problems and solutions. Moscow: Alpha Press.

Saaty, T. (1993). Decision-making. Analytic hierarchy process. Moscow: Radio and Communications.

Sergeev, V. (2001). Logistics in business. Moscow: Infra-M.

Sergeev, V. (2008). Systems and logistics: Information systems and technologies. Moscow: Alpha Press.

Stepanov, V. (2006). Logistics. Textbook. Moscow: Prospectus Publishing House.

Sviridova, O. (2013). Simulation modeling in stochastic problem of inventory management. Economics, statistics and informatics. Bulletin of the EMA, 2, 147-149.

Sviridova, O. (2014). Models to form effective inventory control strategy under uncertainty. Proceedings of the round table on modelling of organizational development. Moscow: Financial University under the Government of the Russian Federation.

Vasiliev, F. (2002). Optimization methods. Moscow: Factorial Press.

\section{Copyrights}

Copyright for this article is retained by the author(s), with first publication rights granted to the journal.

This is an open-access article distributed under the terms and conditions of the Creative Commons Attribution license (http://creativecommons.org/licenses/by/3.0/). 